\title{
Gingival Enlargement Management in Bir Hospital - A Case Series
}

\author{
Dr. Pramod Kumar Koirala, ${ }^{1}$ Dr. Shaili Pradhan, ${ }^{1}$ Dr. Ranjita Shrestha Gorkhali ${ }^{1}$ \\ ${ }^{1}$ Periodontology and Oral Implantology Unit, Department of Dental Surgery, \\ National Academy of Medical Sciences, Bir Hospital, Kathmandu, Nepal.
}

\begin{abstract}
An increase in size of gingiva is a common clinical condition termed as gingival overgrowth. The definite aetiology is unknown. It is classified on the basis of aetiologic factors and pathologic changes. Both localised and generalised overgrowth are encountered commonly and patients are aesthetically, socially, psychologically and functionally disturbed until they revert back to the original contour. Localised gingival enlargement frequently is inflammatory and can also be associated with systemic diseases/condition (hormonal, nutritional, allergic, nonspecific conditioned) or neoplasic and sometimes false enlargement. DIGO is a well documented side effect with the use of anticonvulsant, immunosuppressant, and calcium channel blockers. Total $3 \%$ to $84.5 \%$ of subjects taking these drugs seem to have significant enlargement. Localised overgrowth are managed by proper diagnosis followed by controlling inflammation and other causative factors before surgical excision. DIGO is managed by drug replacement and surgical excision if required after nonsurgical treatment. Gingivoplasty of gingival margin is necessary to create self-cleansing and aesthetic architecture.

Keywords: DIGO; gingival enlargement; gingivectomy; overgrowth.
\end{abstract}

\section{INTRODUCTION}

Gingival enlargement or gingival overgrowth, a common trait of gingival disease, is characterised by an increase in the size of gingiva. ${ }^{1}$ Based on distribution, gingival enlargement can be localised (also called 'reactive lesion of the gingiva' and historically as 'epulis'), regional or generalised. ${ }^{1,2}$ Localised gingival enlargement frequently is inflammatory rather than neoplastic. ${ }^{1}$ Commonly, gingival disease manifests as regional or generalised gingival enlargement which might fall into one of the different types: ${ }^{1}$

\section{inflammatory gingival enlargement;}

gingival enlargement in mouth breathers;

fibrotic (drug induced gingival enlargement; genetic disorders associated with gingival enlargement);

- conditioned gingival enlargement (hormonal; vitamin C deficiency; plasma cell gingivitis)

- gingival enlargement associated with systemic disease: leukemia; Wegener's granulomatosis; Crohn's disease;

\footnotetext{
Correspondence:

Dr. Pramod Kumar Koirala

Periodontology and Oral Implantology Unit,

Department of Dental Surgery, National Academy of Medical

Sciences, Bir Hospital, Kathmandu, Nepal.

email:drpkkoirala@gmail.com

\section{Citation}

Koirala PK, Pradhan S, Gorkhali RS. Gingival enlargement management in Bir hospital - a case series. J Nepal Soc Perio Oral Implantol. 2017;1(2):84-90.
}

sarcoidosis; tuberculous gingival enlargement; multiple myeloma; Hashimoto's thyroiditis; and

- false enlargement.

Gingival hypertrophy could rarely be due to without apparent cause as idiopathic. "Gingival enlargement" or "gingival overgrowth" is the preferred term for all medication-related gingival lesions previously termed "gingival hyperplasia" or "gingival hypertrophy." 3 Though more than 20 prescription medications are associated with gingival enlargement, the drugs associated with gingival enlargement can be broadly divided into three categories: anticonvulsants, calcium channel blockers, and immunosuppressants. ${ }^{3}$ It can be classified on the basis of etiologic factors and pathologic changes. ${ }^{4}$ Localised enlargements could be further divided into three sub-types, viz.,isolated, discrete or regional. "Isolated" enlargements are those limited to gingiva adjacent to single or two teeth (e.g, gingival/periodontal abscess). "Discrete" lesions are isolated sessile or pedunculated, tumour-like enlargements (e.g., fibroma/pyogenic granuloma). "Regional" enlargements refer to involvement of gingiva around three or more teeth in one or multiple areas of the mouth (e.g., inflammatory enlargement associated with mouth breathing in maxillary and mandibular anterior region). "Generalised" enlargement refers to involvement of gingiva adjacent to almost all the teeth present (e.g., drug influenced gingival overgrowth).

Drug induced gingival overgrowth (DIGO) is an adverse effect observed with three types of drugs: phenytoin, an 
antiepileptic; cyclosporine A, an immunosuppressant; and calcium channel blockers, such as dihydropyridines (nifedipine), diltiazem, and verapamil. ${ }^{5}$ The prevalence rate of this disorder has been reported to vary from $10 \%$ to $50 \%$ for phenytoin; ${ }^{5} 8 \%$ to $70 \%$ for cyclosporine $\mathrm{A} ;{ }^{6}$ and $0.5 \%$ to $83 \%$ for nifedipine. ${ }^{7}$ The accurate determination of the prevalence rate in each category is difficult due to the differing indices of gingival overgrowth as gingival inflammation is one reason for difficulty in the accurate assessment of DIGO, because inflammation acts as an exacerbating factor of gingival overgrowth.

Pathogenesis of gingival overgrowth: Although the definite pathology of DIGO is not known, these disorders seem to be induced through the disruption of the homeostasis of collagen synthesis and degradation in gingival connective tissues. It is due to an accumulation of extracellular matrix within the gingival connective tissue, particularly the collagenous component, with various degrees of chronic inflammation. ${ }^{8}$ Although the pharmaceutical effect and primary target tissues of an antiepileptic, an immunosuppressant, and calcium channel blocker are different, they act similarly on fibrous gingival overgrowth. Analysis of the results reveal that the presence of local irritants producing inflammation, gingival enlargement develops after phenytoin administration. In the absence of irritants no enlargement occurs. The cell proliferation and collagen synthesis rates of gingival fibroblasts isolated from human drug-induced overgrown gingiva tend to be greater than those of gingival fibroblasts isolated from nonresponder exposed to nifedipine or phenytoin in-vitro. From these results, DIGO is not due to the increased synthesis of type I collagen but the decreased degradation of type I collagen in gingival connective tissue through the reduction of collagen phagocytosis of fibroblasts. ${ }^{9}$ These drug are known as calcium antagonists. Calcium channel blockers are able to block the influx of calcium ions into cells and to reduce oxygen consumption. Phenytoin is known to act as a calcium channel antagonist and inhibit calcium ion flux. Cyclosporin A is reported to inhibit the release of calcium from intracellular stores, including endoplasmic reticulum and mitochondria. It is thought that integrins transduce information from the extracellular matrix to the inside of the cell by triggering intracellular signaling pathways. Integrin dependent signals have been shown to be essential for cell proliferation, cell response to growth factors and prevention of cell death.

Management strategies: Both localised and generalised overgrowth are encountered commonly and patients are esthetically, socially, psychologically and functionally disturbed until they revert back to the original contour. If there is history of medication, drug replacement is requested by referring to the patient's physician. If required after nonsurgical treatment, they should be treated surgically.
Localised gingival enlargement that has no obvious aetiological basis should be biopsied so that malignant neoplastic disease is identified and treated.

Non- surgical approaches: Non- surgical approach aims to reduce inflammatory component and avoid need for surgery before it affects eating, tooth brushing or appearance. Ideally preventive program before initiation of drug therapies and standard self-maintained oral hygiene in absence of other therapeutic measures is the principle of non-surgical treatment. Meticulous self-administered oral hygiene alongside professionally delivered oral hygiene and scaling and root planing can result in complete resolution or make surgical correction easier. Local environmental factors such as faulty restorations, broken teeth or carious lesions should be eliminated. Any fixed or removable prostheses should be designed to minimize plaque retention.

Drug replacement: New immunosuppressant (e.g. tacrolimus) reduces prevalence and severity to half that of cyclosporin. Cyclosporin concomitant use of calcium channel blockers increase both severity and prevalence. ${ }^{9}$ Ca-Channel Blocker show differential effect. Verapamil substituted for nifedipine and amlodipine. Amlodipine induced gingival hyperplasia is common in Nepal. Angiotensin-converting enzyme inhibitorenalapril \& $\beta$-blocking drug, atenolol are safer. Post-transplant patients receive either azathioprine or cyclosporin to prevent graft rejection. The unwanted effects of Azathioprine on periodontal health of post-renal transplant patients is not reported.

Anticonvulsant substitution: Phenytoin usage now declining due to its adverse effect profile and introduction of new antiepileptics. Changing medication a challenge, and a gradual staged approach is required, may take 2-3 months. Carbamazepine, ethosuximide and sodium valproate are alternatives not to cause DIGO. ${ }^{9}$

Surgical Management: Most frequent treatment of severe overgrowth is surgical management. Use of clinical indices such as gingival overgrowth index more than $30 \%$ needs surgical intervention. It is indicated when there are aesthetic, psychological or functional problem. The main surgical techniques is scalpel gingivectomy. It is the surgical treatment of choice first advocated in 1941 in which soft tissue wall of pocket is excised. Conventionally excess tissue is released by means of a long bevel incision. Complete removal of pocket tissue as a part of excised tissue mass, particularly in inter-dental region is done. Overgrowth flap surgery is to remove enlarged part of gingiva along with pocket reduction therapy when enlargement is associated with bone loss. Electrosurgery and laser excision are alternative to scalpel surgery. Lasers have advantages, but they are expensive and long-term benefits need to be established. Scalpel is quicker to use for both incision and excision than electrosurgery and $\mathrm{CO}_{2}$ laser. 
Adjunctive agents: Regular use of chlorhexidine in phenytoin- induced gingival overgrowth helps to reduce recurrence rate after surgery. But, bacterial resistance and taste disturbance is encountered. Folic acid mouthwash (1 mg/ml) reduce recurrence of phenytoin induced gingival overgrowth. Topical folate may reduce gingival inflammation by binding to plaque-derived endotoxins. Evidence suggest combination of oral hygiene reinforcement and systemic antibiotics may be beneficial. Azithromycin is found to be more effective than metronidazole in changing subgingival biofilm, reducing inflammation and reducing concomitant bacterial infection and hence inflammation.

\section{CASE REPORT 1: Reactive gingival overgrowth in lower labial gingival margin (Figure 1)}

A 45-year-old female patient reported with a chief complaint of intraoral localized painless swelling on the gums at the lower front region lasting for a year. There was no history of trauma, infection, allergy, endocrine abnormality or intake of drugs. No abnormality was detected on extraoral examination and lymph nodes were not palpable. Intraoral examination showed a well demarcated round, soft, sessile, non-tender, non-ulcerated reddish pink swelling on the left side of mandibular anterior gingiva measuring $1 \times 1.5 \mathrm{~cm}$ in diameter. There was no history of tobacco, betel nut chewing or smoking reported by the patient. Oral hygiene status was poor and subgingival deposits were identified. Intra oral periapical radiograph (IOPAR) revealed no abnormal findings. Treatment consisted of oral prophylaxis and excisional biopsy of the gingiva sent for histopathological assessment.

\section{CASE REPORT 2: Reactive gingival enlargement of upper arch (Figure 2)}

A 63-year-old female from eastern part of Nepal presented with localized painless enlargement in upper arch proximal to gingival region of right canine and premolar measuring 1x1 $\mathrm{cm}$ in diameter. Bleeding on minor trauma and discomfort on eating and tooth brushing was experienced. Being a smoker, the patient worried of malignancy. Any systemic disease, medication, or allergy were not seen. Lymph nodes were not
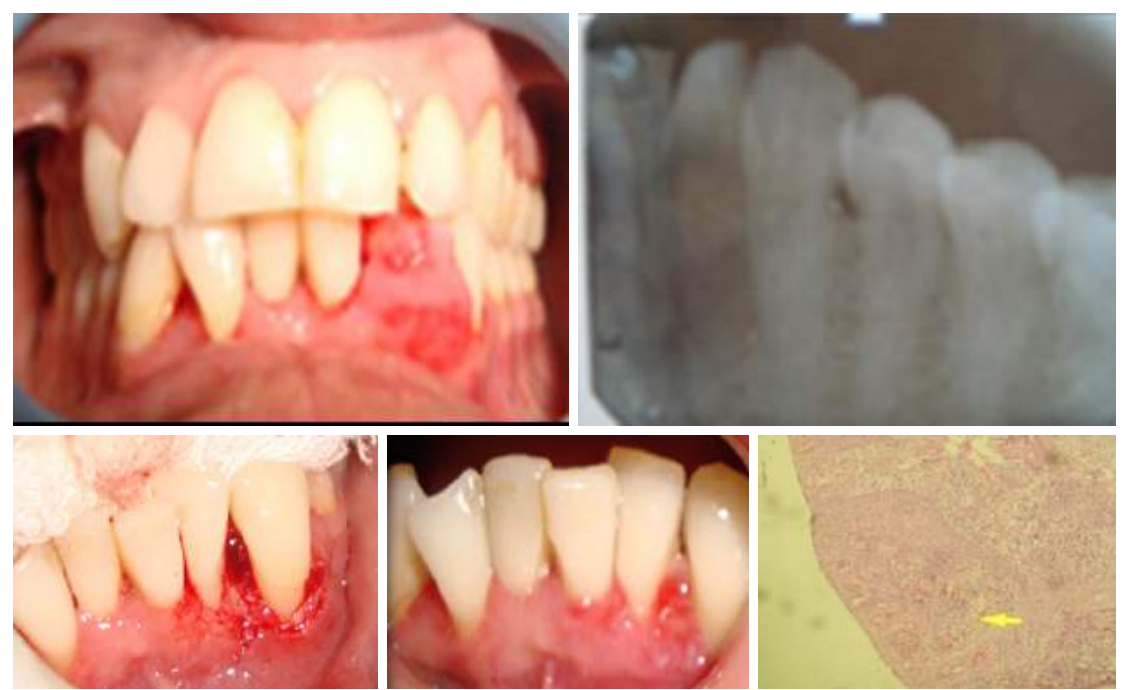

Figure 1: Pyogenic granuloma excision and histopathological picture.
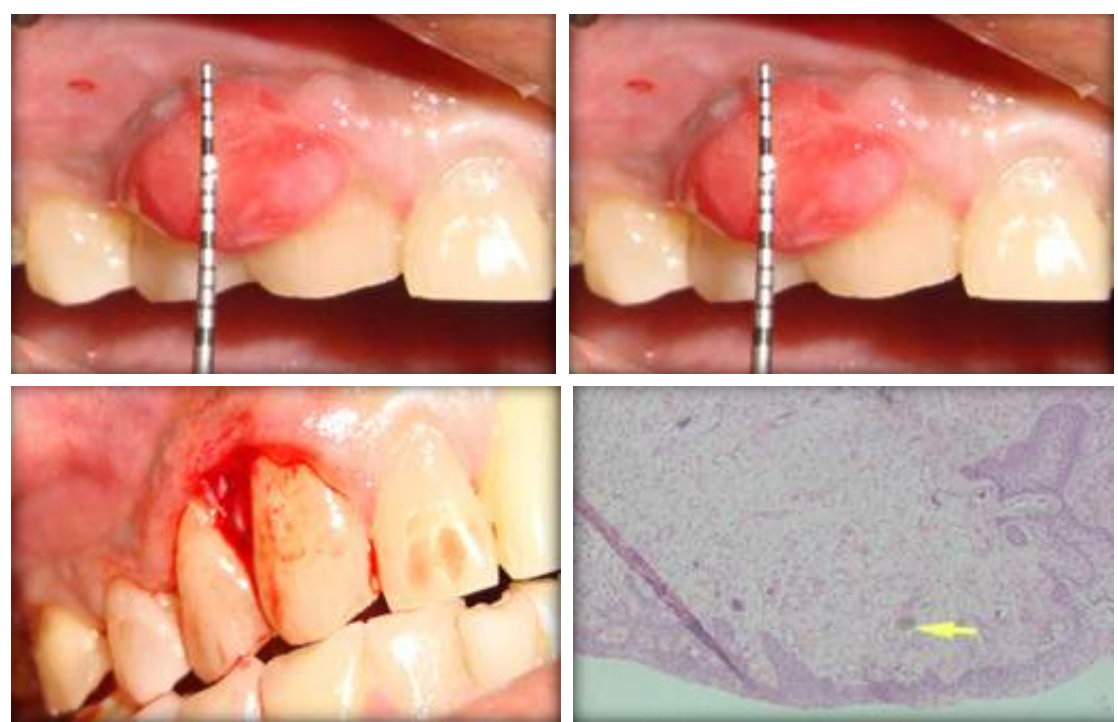

Figure 2: Regression of overgrowth after non surgical therapy clearly demarcated from healthy tissue. 

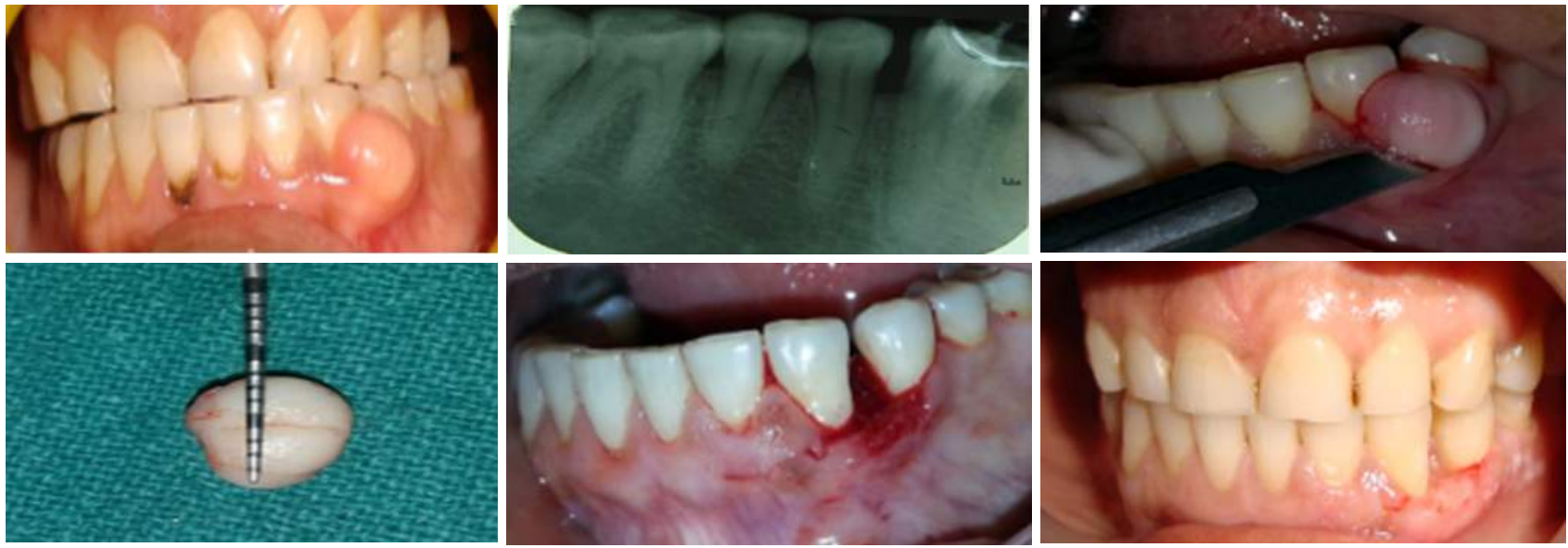

Figure 3: Excision of gingival enlargement (pre and post photographs).

palpable. Erosion of teeth with plaque and calculus deposit was found. IOPAR revealed no abnormal finding. Treatment consisted of smoking cessation counselling and four visits of nonsurgical periodontal therapy. Excisional biopsy of the enlargement sent for histopathological assessment revealed chronic inflammatory lesion with no dysplasia.

Histopathology report: Stratified squamous epithelium with mild acanthosis and pseudo-epithelomatous hyperplasia, fibrocollagenous tissue, edematous stroma along with diffuse chronic inflammatory cell infiltrate, lymphocyte and congested blood vessels in stroma.

\section{CASE REPORT 3: Pregnancy induced gingival overgrowth (Figure 3)}

A 50-year-old female from far western part of Nepal presented with a sessile fibrous overgrowth in lower buccal segment gingiva. History said that the growth persisted for last five years which appeared in her pregnancy. The firm overgrowth was $0.7 \times 0.9 \mathrm{~mm}$ in diameter. Patient was fit otherwise. IOPAR showed spacing between adjacent teeth. The lesion was excised with external bevel incision including healthy gingival margin and sent for histopathological assessment which revealed angiogranulomatous changes compatible with irritation fibroma.
CASE REPORT 4: Amlodipine induced gingival overgrowth associated with plaque deposit (Figure 4)

A 34-year-old male from Bhotahiti, Kathmandu presented with pain and generalised swelling of gingiva with plaque and calculus deposit along with bleeding and oozing of pus from gingival margin. Patient was taking a daily dose of $5 \mathrm{mg}$ of Amlodipine. It was managed by nonsurgical periodontal therapy and drug substitution with Atenolol (beta blocker).

\section{CASE REPORT 5: Amlodipine induced gingival overgrowth surgical treatment after drug substitution and conservative therapy (Figure 5)}

A 48-year-old male reported to the department of dental surgery, perio unit, with chief complaint of enlarged gum in the mouth for last 5 years. He had pain on fractured left lower first molar and was unable to chew normally. He was hypertensive taking $5 \mathrm{mg}$ of Amlodipine daily and Aspirin. He had a habit of smoking. After periodontal and radiographic assessment treatment started with drug substitution and nonsurgical therapy along with extraction of 36. As pain and inflammatory enlargement subsided, surgical therapy was planned, which included internal bevel scalloped incision
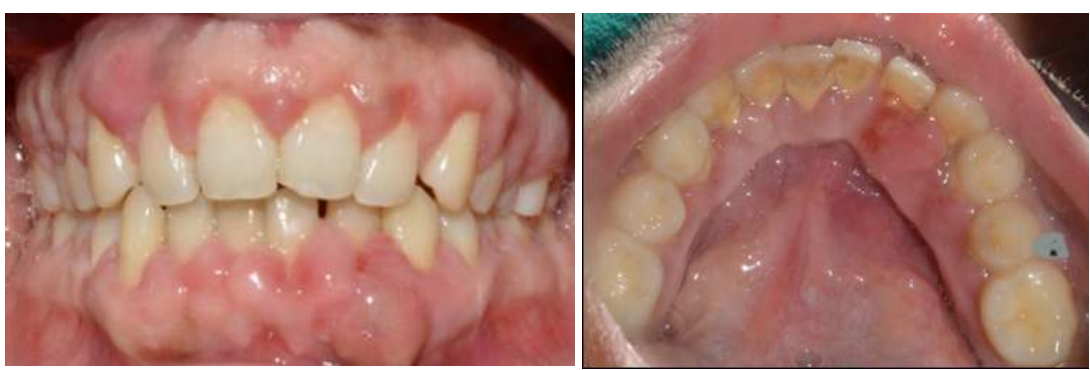

Figure 4: Before and after treatment.

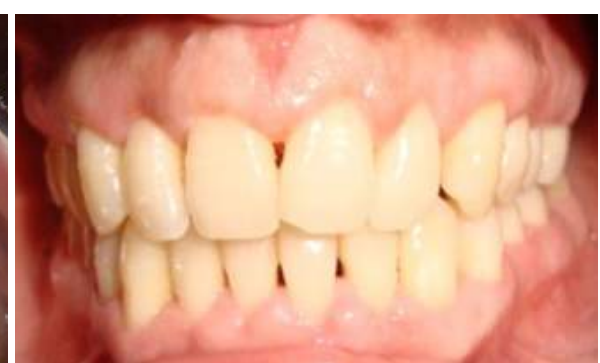

(2) 

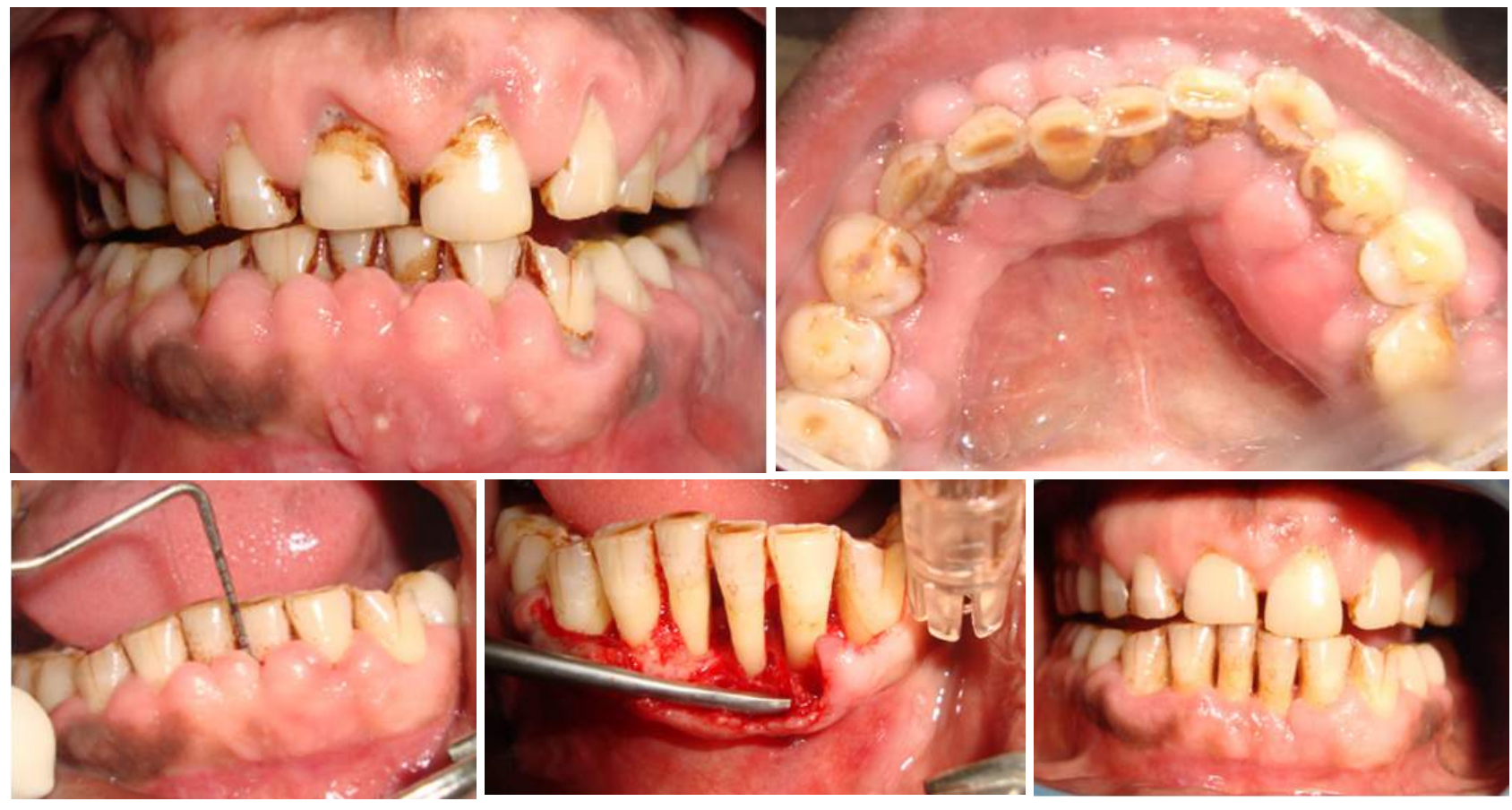

Figure 5: After nonsurgical and surgical therapy (Periodontal pocket depth $(31)=5-6 \mathrm{~mm})$.

and undisplaced flap technique for periodontal pocket reduction in lower anterior incisal region and external bevel gingivectomy where there was no bone loss as it was a combined type of enlargement.

\section{CASE REPORT 6: Hyperplastic gingival overgrowth (Figure 6)}

A 20-year-old female presented with hyperplastic gingival overgrowth developed after decrowding of lower anterior segment which caused an aesthetic and functional problem. She had incompetent lip seal and habit of mouth breathing. External bevel gingivectomy was planned which was carried out with Kirkland knife after few visits of nonsurgical therapy.
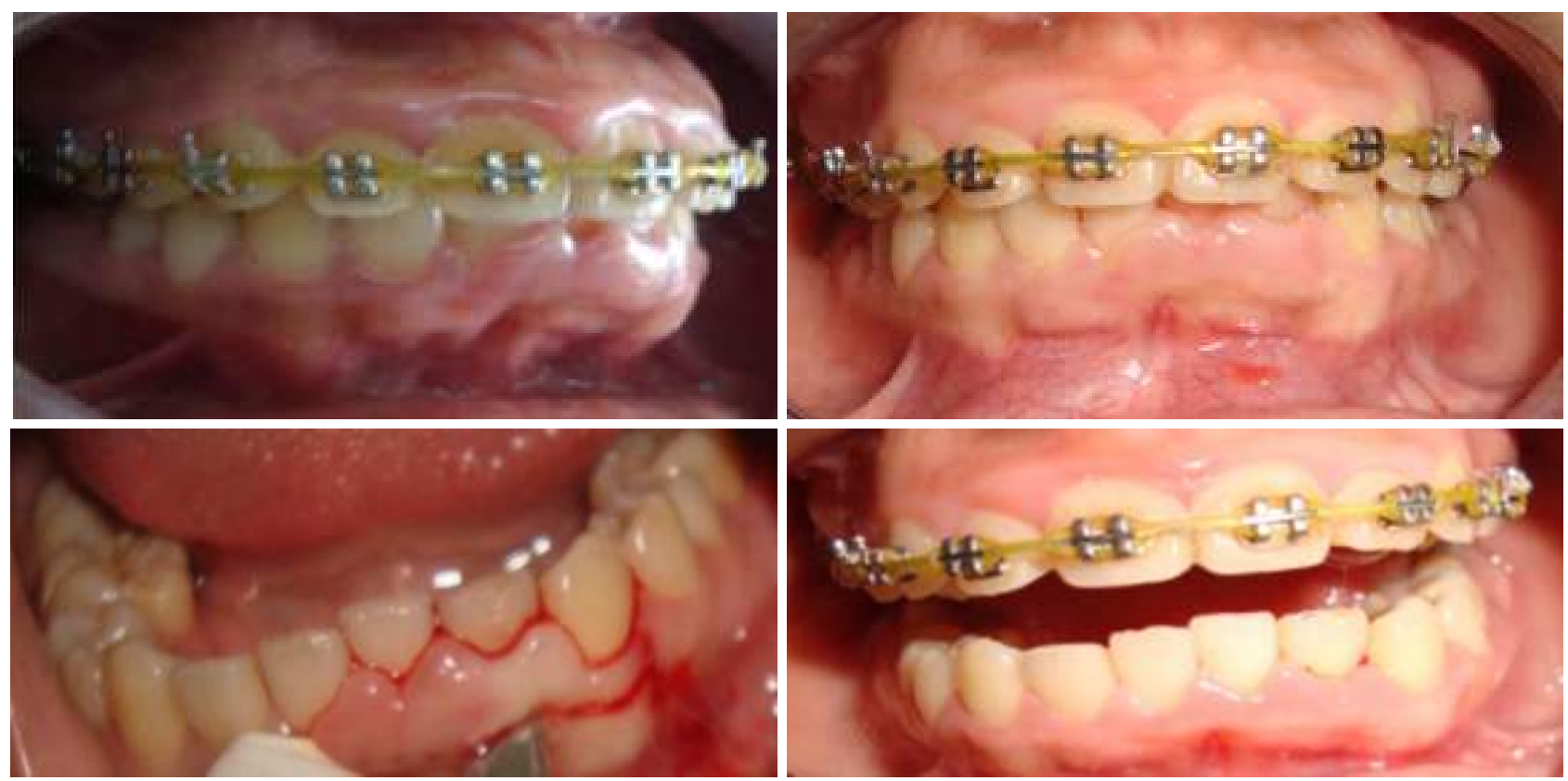

Figure 6: After surgical excision and healing. 


\section{DISCUSSION}

In spite of a myriad of aetiologies, gingival enlargements can often be diagnosed by a careful history (e.g., drug influenced or hormonal influenced gingival enlargement), by location (e.g., mouth-breathing enlargement around anterior teeth) or by the clinical presentation (e.g., strawberry gingivitis). Presence of local irritants (plaque and calculus) could be primary or associated cause of gingival enlargements. Hence, plaque control is an essential aspect of management in all the patients. An excisional/incisional biopsy and/ or hematologic/histological examination may be needed occasionally to correctly diagnose the uncommon cases of gingival enlargements. Differential diagnosis of gingival enlargement requires thorough dental and medical history, careful evaluation of the type, nature and extent of enlargement and identification of etiologic or predisposing factors. Furthermore, laboratory investigations and/ or biopsy specimens may be required to confirm the diagnosis or make a diagnosis of exclusion. The clinician should have an open mind and consider all possibilities before coming to the final diagnosis.

Despite our greater understanding of the pathogenesis of DIGO, its treatment still remains a challenge for the Periodontist due to high recurrence rate and persistence of other risk factors. The primary aim of non-surgical approaches is to reduce the inflammatory component in the gingival tissues and thereby avoid the need for surgery. Ideally preventive programmes should be instituted before the initiation of drug therapies implicated in DIGO. Phenytoin enlargements are uncommon these days with discontinuation of the drug. Carbamazepine, ethosuximide and sodium valproate are alternatives to phenytoin which have shown not to cause DIGO. Among calcium channel blockers, amlodipine has a prevalence of severe gingival overgrowth half that of nifedipine.

The surgical treatment of choice is the gingivectomy, which was first advocated for drug-induced gingival overgrowth in $1941 .{ }^{10}$ The soft tissue wall of the pocket is excised. Perioperative haemorrhage is the main disadvantage of scalpel excision, and this can be significant in highly vascularised and inflamed overgrown gingival tissues. Electrosurgical techniques have been used in dentistry for the past 70 years. Although such techniques produce adequate haemostasis, they have the disadvantage of causing a surrounding zone of thermal necrosis, which may impede wound healing. Reports in the literature have confirmed delayed healing of electrosurgery wounds when compared with scalpel wound healing. ${ }^{11}$ Flap surgery may be complicated by excessive gingival enlargement, especially in the inter-proximal space. It would seem to be more suited for those cases of mild to moderate overgrowth associated with both bone and attachment loss.

The dental lasers may be another useful alternative treatment to conventional gingivectomy techniques. The use of laser surgery to remove excess gingival tissue has been described by a number of authors. ${ }^{11}$ Lasers have remarkable cutting ability and they also generate a coagulated tissue layer along the wall of the laser incision which promotes healing.

Cases of localised or generalised gingival overgrowth are common presentations which are encountered in everyday practice. The localised reactive enlargement, which are chronically inflamed and are associated with local and systemic factors, are commonly encountered. Mouth breathers and drug induced gingival enlargement cases are common too. Management of any sort of extra growth is compelling and control of inflammation by meticulous cleaning and oral hygiene maintenance makes the procedure easy and decreases the recurrence. All patients about to be medicated should go through a full periodontal assessment and treated appropriately. Cyclosporin, amlodipine, and phenytoin therapy cause an increase in gingival overgrowth, which may be related to plasma concentrations of the drug. Proper assessment, diagnosis and eradication of predisposing factors before excision \& confirmation of diagnosis with histopathology is key to successful management.

\section{ACKNOWLEDGEMENT}

We would like to acknowledge Dr. Sujaya Gupta, Dr. Binod Adhikari, Dr. Sweta Shrestha, Dr. Shweta Agrawal and Dr. Rejina Shrestha. 


\section{REFERENCES}

1. Agrawal AA. Gingival enlargements: Differential diagnosis and review of literature. World J Clin Cases. 2015 Sep 16;3(9):779-88.

2. Lee K. The fibrous epulis and related lesions. Granuloma pyogenicum,'Pregnancy tumour', fibro-epithelial polyp and calcifying fibroblastic granuloma. A clinico-pathological study. Periodontics. 1968 Dec;6(6):277-92.

3. Dongari-Bagtzoglou A, American Academy of Periodontology. Academy Report. Informational paper: drug-associated gingival enlargement. J Periodontol. 2004;75(10):1424-31.

4. Newman MG, Takei H, Klokkevold PR, Carranza FA, editors. Carranza's Clinical Periodontology - A South Asia Edition. 11th ed. New Delhi, India: Saunders, Elsevier; 2011.

5. Nishikawa S, Nagata T, Morisaki I, Oka T, Ishida H. Pathogenesis of drug-induced gingival overgrowth. A review of studies in the rat model. J Periodontol. 1996 May;67(5):463-71.

6. Seymour RA, Heasman PA. Drugs and the periodontium. J Clin Periodontol. 1988 Jan;15(1):1-16

7. Barak S, Engelberg IS, Hiss J. Gingival hyperplasia caused by nifedipine: Histopathologic findings. J Periodontol. 1987 Sep;58(9):639-42.

8. Yamasaki A, Rose GG, Pinero GJ, Mahan CJ. Ultrastructure of fibroblasts in cyclosporin A-induced gingival hyperplasia. J Oral Pathol. 1987 Mar;16(3):129-34.

9. Kataoka M, Kido J, Shinohara Y, Nagata T. Drug-induced gingival overgrowth--a review. Biol Pharm Bull. 2005 Oct;28(10):1817-21.

10. Thomason JM, Ellis JS, Jovanovski V, Corson M, Lynch E, Seymour RA. Analysis of changes in gingival contour from three-dimensional co-ordinate data in subjects with drug-induced gingival overgrowth. J Clin Periodontol. 2005 Oct;32(10):1069-75.

11. Liboon J, Funkhouser W, Terris DJ. A comparison of mucosal incisions made by scalpel, CO2 laser, electrocautery, and constant-voltage electrocautery. Otolaryngol Head Neck Surg. 1997 Mar;116(3):379-85. 